\title{
On the Performance Degradation of Centrifugal Pumps
}

\author{
A. Eaton, F. D'Alessandro, W. Ahmed, M. Hassan \\ University of Guelph \\ 50 Stone Rd E, Guelph, Canada \\ eatona@uoguelph.ca; fdalessa@uoguelph.ca; ahmedw@uoguelph.ca; mahassan@uoguelph.ca
}

\begin{abstract}
In this paper, a review on the performance degradation of centrifugal pumps is presented. Pump performance characterization is discussed as well as the common degradation affecting pump operation is summarized. Additionally, the methods used to determine the effect of degradation on the pump performance over its lifetime is discussed. It was concluded that in order to develop a better understanding of performance degradation, a deliberate testing program that controls degradation mechanisms while continuously records pump performance is required. Some of the models found to measure performance degradation such as the Cox Process Regression Model can be used to develop an appropriate prediction tool for performance degradation.
\end{abstract}

Keywords: Centrifugal Pump, Pump Performance, Prediction, Acceleration, Degradation.

\section{Introduction}

One of the most prominent issues in today's society is decreasing the impact that humans have on the environment. As the human population continues to increase, the global energy demand will follow a similar trend. Centrifugal pumps account for approximately $20 \%$ of the global energy demand [1]. Furthermore, the operation costs associated with running the pump typically outweighs the initial cost of the pump itself. The study of degraded centrifugal pump performance is critical to improve the operation of various industrial processes including power production, water distribution, agriculture, heating, cooling and food processing. Additionally, for successful energy auditing of buildings and industrial facilities the operational state of the of the pumps should be taken into account. With centrifugal pump efficiencies in the range of 60-90 percent depending on capacity, the long term costs due to the inefficiencies can become significant over the lifetime of the pump. With highly reliable products, which is the case for centrifugal pumps, little information about the change in performance is known during the pump operation until the substantial drop in performance effects the overall system process to which the pump is driving [2]. A prominent cause of a decrease in pump performance can be attributed to the degradation of the pump itself, yet there has been no adequate relationship developed between the two [3]. This paper explores how to approach modelling this relationship.

There are two approaches used to directly measure the performance of a pump. The first method is considered conventional and consists of the direct measurement of pump flow rate $(\mathrm{Q})$, the pump head $(\mathrm{H})$, and input power to the pump shaft $[1,4]$. The pump efficiency can be calculated using the following equation.

$$
\eta_{p}=\frac{\rho g Q H}{\tau \omega}
$$

Where, $\tau$ is the torque applied to the pump shaft and $\omega$ is the measured rotational speed. Measuring the torque and rotational speed of the pump shaft allows for a direct measurement of the input power to the pump impeller and thus an accurate representation of the efficiency. This method relies heavily on an accurate flow rate measurement, which can be difficult and expensive to obtain in the field [4]. An inaccuracy of such measurements can lead to large errors in Equation 1 , and unreliable performance evaluations.

The second method uses a thermodynamic analysis to measure the thermal gain of the pumped fluid. The losses within the pump are transferred into thermal energy, which is then absorbed by the water that is being pumped. The efficiency can be expressed by the following equation [4]. 


$$
\eta_{p}=\frac{E_{H}}{E_{M}}
$$

where, $E_{H}$ is the hydraulic energy per unit mass of liquid and $E_{M}$ is the mechanical energy per unit mass and can be determined using the equations below.

$$
\begin{gathered}
E_{H}=\frac{\Delta P}{\rho} \\
E_{M}=a \Delta P+c_{p} \Delta T
\end{gathered}
$$

where, $\Delta P$ is the pressure difference across the pump, a is the isothermal coefficient (change of enthalpy with pressure at constant temperature), $c_{p}$ is the specific heat capacity and $\Delta T$ is the differential temperature across the pump [4]. This method, although relatively new, yields high accuracy under the proper conditions (low concentration of particulates and high flow rates).

To determine the full operational range of the pump's performance an H-Q curve is constructed at a fixed impeller geometry and fixed rotational speed. To get the full representation of the pumps operational capacity a complete set of $\mathrm{H}-$ Q curves should be developed by generating a curve for a variety of rational speeds and impeller geometries.

\section{Degradation in Centrifugal Pumps}

Operational and design decisions can have a negative impact on the performance of a centrifugal pump. Pump surge can occur due to a rapid valve closure, hard pump start up, sudden stoppage or during the removal of air pockets. An uncontrolled pump surge can lead to failure of the pump and other components of the piping system [1]. Furthermore, impeller suction recirculation can cause cavitation and surface pitting as a result of vortex formation that attaches to the vane system [1]. A similar degradation mechanism occurs on the discharge side of the impeller. Both forms of recirculation occur due to the pump operating at very low flow rates. Fluid viscosity can also decrease rate of degradation if the pump is not properly selected for the application [1].

Solid particle erosion has been extensively examined particularly with the prediction of erosion rates and pump head drop as a result of slurry pumping. Erosion rates are dependent on the characteristics of the carrier fluid, the concentration of solid particles in the slurry, the physical properties of the solid particle, the flow conditions (velocity, impact angle) and the characteristics of the target material (surface roughness, hardness, chemical composition) [5]. The results of their review indicated that the research pertaining to the erosive wear of pump materials was primarily done in an external test rig with material samples rather than the pump components [5]. The main methods of experimentally testing erosion wear was done in a pot tester, which was then followed by the jet impingement tester, air jet tester, and Coriolis tester. It is concluded that the majority of research regarding erosion wear lacks information when surface roughness of the target material is varied. Furthermore, erosion rates at elevated temperatures needs investigation [5]. A review of the hydraulic performance and erosive wear in centrifugal slurry pumps is presented by [6]. With respect to the hydraulic performance of the pump the review is mainly concerned with the decrease in pump head due to the added weight of the solid particles. Wear rates due erosion mechanisms was discussed in depth. Many of the research efforts to test erosion wear is performed using a paint/epoxy layer applied to various pump components (impeller and casing). This method was useful in determining the location of erosive wear in the pump as well as relative erosion rates when compared to less affected components [6].

Corrosion in centrifugal pumps can be uniform or it can be very localized affecting only a small region of a pump component [7]. Uniform corrosion typically occurs due to the absence of a protective film on the entire surface and can be accelerated by the addition of oxygen and a decrease in the $\mathrm{pH}$ [8]. Furthermore, the electrochemical reaction between a susceptible metal and the water forms an oxide layer that is visible in the form of rust on the surface of the component. This form of corrosion is preferred compared to localized forms as it is predictable and can often be controlled by selecting proper materials. Localized corrosion mechanisms can be broken down into six separate processes. First, galvanic corrosion occurs when two dissimilar metals are in contact and are exposed to an aqueous environment [7]. The contact allows for the transfer of electrons from one metal to another and as a result induces material wear. The 
conductivity of the water will have a large impact on the corrosion rate. Pitting corrosion occurs as a result of a small hole in the protective film and the formation of a small anodic region. With such a small anodic region, the small hole continues to get deeper until failure or replacement of the component. It should be noted that pitting corrosion is most commonly found in various stainless steel alloys that contain a protective chrome oxide layer [7]. Less common forms of localized corrosion include graphitic, dealloying, crevice and under-deposit corrosion. These mechanisms are primarily due to the microstructure of the metal and is discussed in more detail in [7].

In order to investigate the performance of a pump over the course of its lifetime, the pump must be exposed to wear accelerating conditions to collect data in a shorter period of time which brings rise to the following section.

\section{Accelerated Degradation Test (ADT)}

Accelerated degradation testing is concerned with statistical models and data analyses concerning the degradation of product performance over a predetermined time period at using design and overstressed conditions. This is done by using statistical models to extrapolate performance degradation data to estimate a time when performance reaches a predetermined failure level or similarly, when the performance falls below a specified performance threshold. Such extrapolation allows one to examine the effect on life of different design choices or assumptions about the performance resulting in failure [9]. However, choosing a suitable model and appropriate definition of failure in terms of performance is needed. The following section describes suitable models for performance in centrifugal pumps.

One approach to ADT modelling is to assume that the degradation is a stochastic process in time, with the consideration of only one random parameter [9], as seen with the Wiener Process Regression Model [10]. An alternative approach is to consider general models such as the Cox Process Regression Model [3], where multiple random variables contributing to performance are considered. Both models have been described in Table 1. The Wiener Process has found application as an appropriate model to predict a future degradation level or prediction of lifetime in other studies [11, 12]. The Cox Model has also been extensively used in degradation modelling seen in [13, 14].

Since a regression model is being used, the distribution of data will also be estimated. Again, one of two approaches can be taken. First, by using parametric methods, seen with the Birnbaum-Saunders Distribution [15] which was developed to model the distribution of metal fatigue; and secondly, by using a nonparametric method such as the Weibull Distribution [9]. By definition, nonparametric methods include the assumption that the data does not conform to a well studied distribution, and the distribution has the ability to assume the characteristics of many different types of distributions, but are heavily dependent on the sample size [11]. The Weibull Distribution is extensively used in reliability engineering and failure analysis [8] and can accurately describe fatigue test results [14].

Table 1: Comparison of ADT models.

\begin{tabular}{|c|c|c|}
\hline & Wiener [16] & Cox [17] \\
\hline Model Equation & $W_{n}(t)=\frac{1}{\sqrt{n}} \sum_{1 \leq j \leq[n t]} \varepsilon j$ & $h_{x}(t)=h_{o}(t) e^{\sum_{i=1}^{n} \beta x}$ \\
\hline Description & $\begin{array}{l}\text { Basis of model: Using Brownian } \\
\text { Motion as a limit of random walks to } \\
\text { progress toward lower limit of the } \\
\text { performance threshold } \\
\text { - where } \varepsilon_{1}, \varepsilon_{2} \text { are a sequence of } \\
\text { independent and distributed random } \\
\text { variables } \\
\text { Where } n \text {, is number of intervals } \\
\text { of degradation and } n>1 \text { defines a } \\
\text { continuous time-stochastic process. }\end{array}$ & $\begin{array}{l}\text { - Developing a distinction } \\
\text { between degradation rate and } \\
\text { proportion of influential variables } \\
\text { f } h_{x}(t) \text { is the performance } \\
\text { function that is dependent on the } \\
\text { baseline performance, } h_{o}(t) \text {, and the } \\
\text { impact }(\beta) \text { on performance for each } \\
\text { variable, } x \text {. }\end{array}$ \\
\hline
\end{tabular}

\section{ADT Models Evaluation}

Recently, in Canada, degraded pump performance was investigated in 150 pumps ranging from $30 \mathrm{Hp}$ to $4000 \mathrm{Hp}$ using thermodynamic methods. On average, the pump efficiency deviated by $9.3 \%$ measured across when the pump is operating at the best efficiency point. Additionally, the efficiency dropped $12.7 \%$ when the actual operating point 
and the original best efficiency point are compared [18]. Papa [18] looked at pumps that had been operating in the field and the performance was measured at one instant in time. The performance over the lifespan of the pump needs to be investigated to obtain a better understanding of how degradation correlates to pump life and pump efficiencies at various operating points. A method to determine pump performance over time is developed by [19]. By modelling the leakage flow due to wear ring degradation as flow through a pipe, the performance curve of a degraded pump can be determined. By taking the hydraulic resistance at an associated pipe diameter to be constant, a relationship between the original H-Q curve and the degraded H-Q curve is derived. The model is justified based on the prediction of a small difference in head at near pump shut off head when compared to a new pump however, lacks experimental validation. It was found that one of the main methods to monitor the pump performance over time is to record the head produced by the pump at a given flow rate as described by [2]. Figure 1 depicts a sample trend of head reduction in centrifugal pumps over the course of its lifetime. Similar to what was shown in [2], the reduction in head increases as a non-linear relation. Using methodologies behind accelerated degradation testing, the reduction the same head reduction

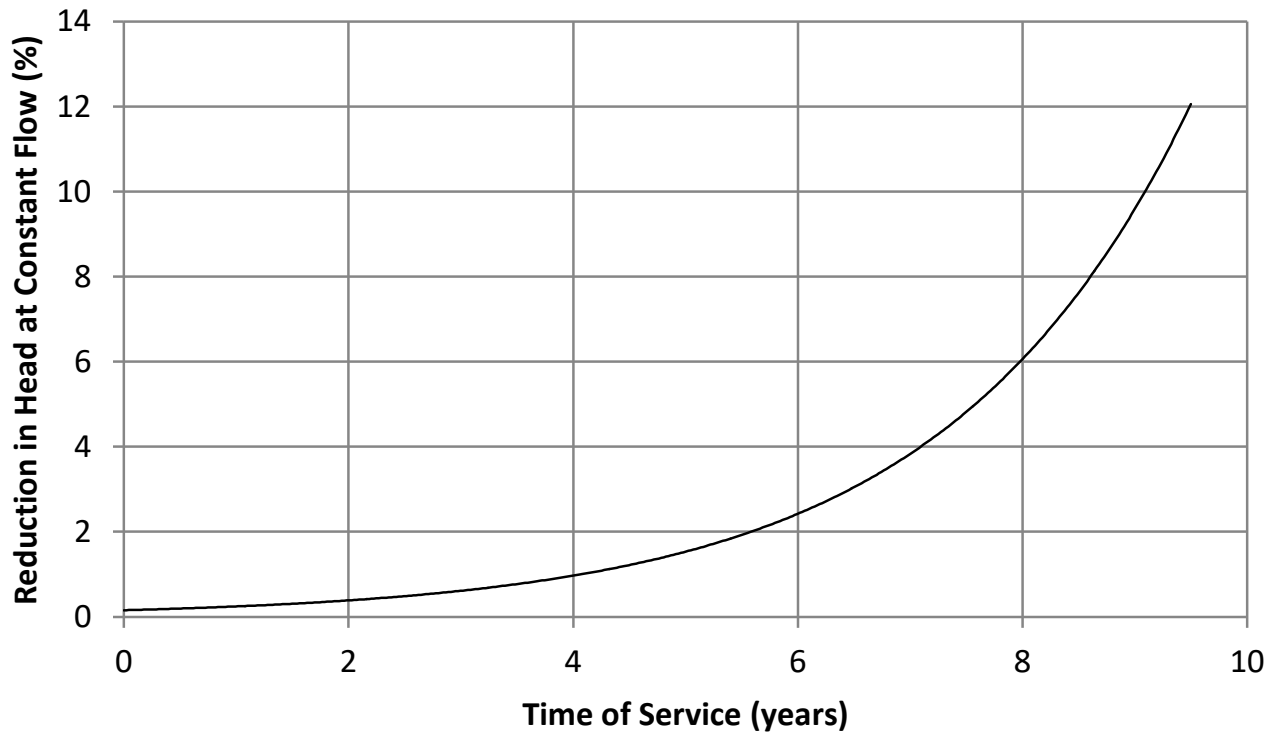

Fig. 1: Degradation of pump performance shown by the reduction of head at a constant flow rate.

will be seen in a fraction of the time. Accelerated performance decay similar to what is shown in Figure 1 is discussed by [20]. The proposed model is based upon the initial performance of a product and degraded data and can be seen in the following equation.

$$
P(t)=P^{N}-b t^{c}
$$

where, $\mathrm{P}(\mathrm{t})$ is the performance of the product at time, $\mathrm{t}, \mathrm{P}^{\mathrm{N}}$ is the initial performance, $\mathrm{b}$ and $\mathrm{c}$ are constants that are solved for using the least square method for curve fitting [20]. This approach is valuable as it takes numerous data points of a product subjected to the same wear mechanism and creates a curve that can be used to estimate the performance at any point in the products lifetime.

There are also various methods to indirectly monitor the working condition of a centrifugal pump with respect to the level of degradation. The first and commonly employed method is vibration analysis. Increase in vibration amplitude and changes in the frequency measurements can indicate impeller unbalance, misalignment, and looseness of vibrating components. Sampling of lubricants for material wear debris can be helpful for accessing bearing wear within the motor and shaft assembly. Electrical consumption by the motor can also indicate pump degradation, as more power will be used for the same demand from the pump. However, this may also account for motor degradation [2].

Due to the unknown correlation between the four performance variables, pump degradation is categorized as a stochastic process. The relationship between the operating conditions, the age and degradation will be determined by 
using the aforementioned statistical models. The Wiener Process, being a single variable model does not account for the four performance factors, and a "pseudo-variable" correlating such factors will need to be determined from experimental data if used. Assuming all factors that affect the pump performance are uncorrelated, as seen with [21], a Cox Process Regression Model [3] should be used.

Since little research has been done using ADT to determine performance degradation, the expected distribution is undetermined. Although both distributions hold merit for this application either model can produce erroneous results by imposing the constraints of the model itself, for example, improving accuracy by using a larger sample size [22] or assuming the shape of a specific distribution seen in [15]. After experiments have been conducted, a sensitivity analysis pertaining to distribution will be conducted and the appropriate distribution model will be selected.

\section{Conclusion}

There has been ample research dedicated to formulating the methods to measure pump performance. However, lifetime pump performance is rarely touched upon in literature. In order to develop a better understanding of performance degradation, a deliberate testing program that controls wear mechanism (operating conditions, erosion, corrosion, poor pump selection, lack of repairs and maintenance) would be required and which was beyond the scope of the work reported herein. Damage affecting the impeller cannot be directly observed while in operation but the influence on performance can be. To measure the reliability of performance a degradation analysis using Cox Process Regression Model [3], with the application of a sensitivity analysis to determine the appropriate distribution model should be implemented.

\section{Acknowledgement}

The authors would like to acknowledge the support of Natural Sciences and Engineering Research Council of Canada (NSERC). The support of Xylem AWS is greatly appreciated.

\section{References}

[1] H. M. Badr and W.H. Ahmed, Pumping Machinery Theory and Practice. John Wiley \& Sons, Ltd, 2015.

[2] R. Beebe, "Condition Monitoring Methods for Pumps," Chemical Engineering, vol. 119, no. 9, pp. 34-39, 2012.

[3] D. R. Cox, "Some Statistical Methods Connected With Series of Events," J. Royal Statistical Society., vol. 17, no. 2. pp. 121-164, 1955.

[4] S. Cartwright and B. Eaton, "Investigating Energy Savings in Pumps and Pumping system by the Thermodynamic Method," in Pumps: Maintenance, Design and Reliability Conference, 2009.

[5] S. R. More, D. V. Bhatt and J. V. Menghani, "Recent Research on Erosion Wear-An Overview," in Proceedings of 5th International Conference of Materials Processing and Characterization, 2016.

[6] R. Tarodiya, B. K. Gandi, "Hydraulic performance and erosive wear of centrifugal slurry pumps-A review," Powder Technology, vol. 305, pp. 27-38, 2017.

[7] F. Gall, "The Corrosion of Centrifugal Pumps in Aqueous Environments," in Proceedings of Pumps and Compressors Conference, 2013.

[8] J. F. Gulich, Centrifugal Pumps. Springer, 2010.

[9] W. Nelson, Accelerated Testing. Hoboken, NJ: Wiley, 2004.

[10] W. Kahle, "The Wiener Process as a Degradation Model: Modeling and Parameter Estimation," in Advances in Degradation Modeling, M. S. Nikulin, New York, NY: Birkhäuser Boston, 2010, pp. 127-146.

[11] K. A. Doksum and A. Hoyland, "Models for variable-stress accelerated life testing experiment based on a Wiener process and the inverse Gaussian distribution," J. Techno- metrics., vol. 34, pp 74-82, 1992.

[12] J. Lu, "A Reliability Model Based on Degradation and Lifetime Data," Ph.D. Thesis, McGill Univ, Montreal, Canada.

[13] W. Kahle and H. Wendt, "Parametric Shock Models," in Advances in Degradation Modeling, M. S. Nikulin, New York, NY: Birkhäuser Boston, 2010, pp. 77-104.

[14] F. Haghighi, N. Nooraee, and N. R. Rad, "On the General Degradation Path Model," in Advances in Degradation Modeling, M. S. Nikulin, New York, NY: Birkhäuser Boston, 2010, pp. 147-155. 
[15] V. Leiva, "The Birnbaum-Saunders Distribution," London, Academic Press is an imprint of Elsevier San Diego, CA, 2016.

[16] T. Szabados, "An elementary introduction to the Wiener process and stochastic integrals," Studia Scientiarum Mathematicarum Hungarcia, 2010.

[17] D. R. Cox, "Regression Models and Life-Tables," J. Royal Statistical Society, vol. 34, no. 2, pp. 187-220, 1972.

[18] Papa et al., "Pump energy efficiency field testing and benchmarking in Canada," J. Water Supply: Research and Technology, vol. 60, no. 7, pp. 570-577, 2014.

[19] D. C. Gaiewski, "A methodology for determining degraded pump performance based on in-service test criteria or data," in Proceedings of the ASME Power Conference, Denver, CO, 2011.

[20] S. H. Mohammadian, D. Ait-kadi, F. Routhier, "Quantitative accelerated degradation testing: Practical Approaches," Reliability Engineering and System Safety, vol. 95, pp. 149-159, 2010.

[21] J. E. Korenchan, "Application of AnalyticalCenytifugal-Pump Performance Models in Two-Phase Flow," M.S. Thesis, Dept. Mechanical Eng., MIT., Cambridge, Massachusetts.

[22] G. A. Whitmore and F. Schnekelberg, "Modelling Accelerated Degradation Data Using Wiener Diffusion with A Time Scale Transformation,” J. Lifetime Data Analysis., vol. 3, pp. 27-45, 1997. 